\title{
Evenness Preserving Operations on Musical Rhythms
}

\author{
Francisco Gomez-Martin* $\quad$ Perouz Taslakian ${ }^{\dagger} \quad$ Godfried T. Toussaint ${ }^{\dagger}$
}

\begin{abstract}
In this paper we define four operations on musical rhythms that preserve a property called maximal evenness. The operations we define are shadow, complementation, concatenation, and alternation. The proofs of the theorems are omitted from this abstract.
\end{abstract}

\section{Introduction}

A musical rhythm is a repeating pattern of onsets (sounded pulses) that occur at irregular intervals. These patterns have been studied by mathematicians, as well as musicians, for centuries. Perhaps the first mathematician to have noted a connection between these two fields is Pythagoras (6th century B.C), who noticed that the ratio between a string and the frequency of the tone it makes when plucked remains constant as the length of the string is varied [3].

Rhythms can be be seen as two-way infinite binary sequences [9], where each bit represents one unit of time called a pulse; a 1-bit represents a played note or onset and a 0-bit represents a silence (for example, a sixteenth rest). We will use ' $x$ ' for ' 1 ' and ' . ' for ' 0 '. It is generally assumed that the twoway infinite bit sequence is periodic with some period $n$. A representation that better captures this cyclic nature of rhythms is that of a polygon inscribed in a circle called a cyclic polygon. Consider a circle with $n$ points placed at equal distances around its circumference. A $k$-sized subset of these points represents a rhythm with $n$ pulses and $k$ onsets. Connecting pairs of points consecutively along the circumference gives us a cyclic polygon.

A musical piece may contain different rhythms and melodies throughout its progression. What musicians often like to do however is to stay within a theme during this progression. For example, a jazz soloist must respect the style and feeling of the piece, and thus play an improvised variation based on the foundation of the main theme [4]. A way of realizing such an improvisation is by taking the base rhythm and transforming it to another through one or more operations. An operation transforms one musical rhythm to another based on a set of rules. Operations are important for music composition and especially for improvisations. Thus, studying operations is useful both for theoretical analysis as well as providing formal rules for improvisation techniques. Here we study four operations that preserve a property of rhythms called maximal evenness. The operations we study are: shadow, complementation, concatenation, and alternation.

\section{Definition and Notation}

Let $R$ be a rhythm with $n$ pulses and $k$ onsets. When $R$ is represented as a cyclic polygon, the Euclidean distance between two onsets $i$ and $j$ is the length of the chord determined by $i$ and $j$. We say that $R$ is maximally even if the sum of all pairwise Euclidean distances between onsets on the circle is maximized. We distinguish between two rhythms $R$ and $R^{\prime}$ that differ by a rotation of the pulses and

\footnotetext{
*Departamento de Matemática Aplicada, Universidad Politécnica de Madrid, Madrid, Spain, fmartin@eui.upm.es

${ }^{\dagger}$ School of Computer Science, The Schulich School of Music, McGill University \{perouz,godfried\}@ cs.mcgill.ca
} 
onsets. A Euclidean rhythm $E(k, n)$ with $n$ pulses and $k$ onsets is a maximally even rhythm that can be generated by the EUCLIDEAN algorithm [2] that we reproduce below:

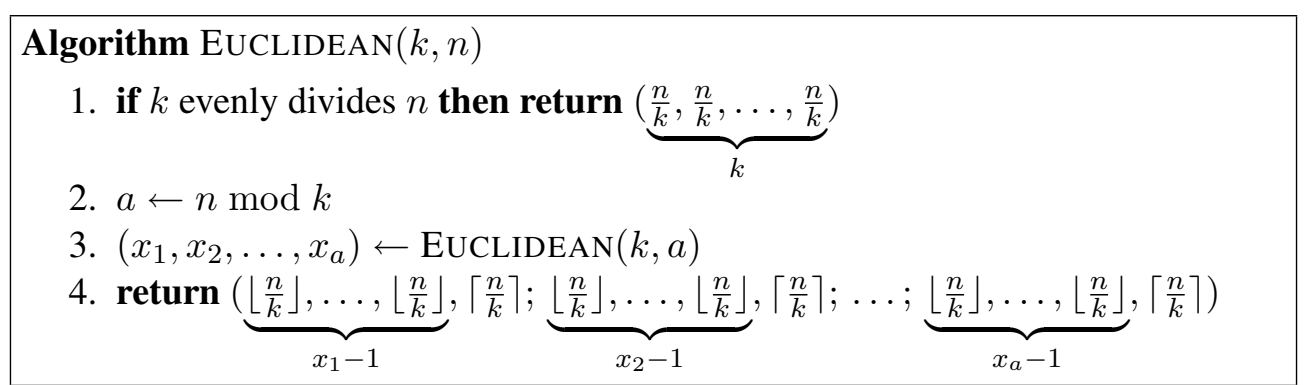

\subsection{Shadow}

Several ethnomusicologists have argued that African drumming, handclapping, and mallet performance is best understood as a motor activity. For example a hand (arm) is raised and then dropped to strike the instrument. According to Jay Rahn [7], one possible mechanism for the tacit motor mediation of attack points of onsets is the peaking of the gesture at the temporal midpoint between two sounds. He calls the sequence of midpoints of the onsets of a rhythm the shadow of the rhythm. For example the Cuban tresillo rhythm given by $[\times \ldots \times \times \ldots \times \ldots$ has the shadow $[\cdots \times \cdots \times \times \cdots \times \cdot]$, which yields the shadow rhythm $[\times \cdots \times \cdots \times \cdots \times$ (Figure 1$)$.

Performed on a rhythm, the shadow operation increases the even-

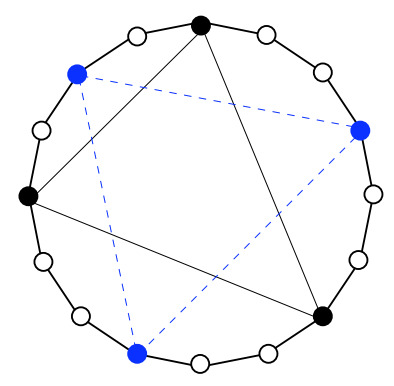

Figure 1: The Cuban tresillo and its shadow.

ness of the new rhythm. One question that comes to mind is: what happens to the sequence obtained when we continue to perform this operation on every rhythm resulting from the shadow of another? It turns out that this is a geometric problem that has been investigated in the mathematics literature.

The study of properties of sequences of polygons generated by performing iterative processes on an initial polygon $P^{0}$ has received much attention, and the shadow operation is just one of many operations that has been investigated. Perhaps the most studied sequence is the one sometimes referred to as Kasner polygons [8]. Given a polygon $P^{0}$, the Kasner descendent $P^{1}$ of $P^{0}$ is obtained by placing the vertices of $P^{1}$ at the midpoints of the edges of $P^{0}$ (making $P^{1}$ the shadow of $P^{0}$ ).

Let $P^{0}, P^{1}, P^{2}, \ldots$ be a sequence of cyclic polygons such that the vertex set of $P^{i}$ is the shadow of the vertex set of $P^{i-1}$ for all integers $i \geq 0$. We say that $P^{i}$ is the shadow polygon of $P^{i-1}$. Hitt and Zhang [5] show that given any convex cyclic polygon $P^{0}$, its shadow sequence converges to a regular polygon. From their proof, it follows that the area of each $P^{i}$ is greater than or equal to the area of $P^{i-1}$ for any $i>0$, with equality only when $P^{i}$ is regular.

On a circular lattice such as $R$, a vertex $a_{i}$ of the shadow might not lie on a lattice point (a pulse) thus violating the definition of a rhythm. To avoid this problem, in such a case we move $a_{i}$ to the nearest lattice point in the clockwise direction. The result is the discrete shadow of $R$. We now can show the following:

Theorem 1 The discrete shadow of a Euclidean rhythm $R$ is a rotation of $R$.

\subsection{Complement}

The complement of a subset $R$ of a set of elements is the set of all elements that are not in $R$. Consequently, the complement of a rhythm $R$ with $k$ onsets is the rhythm having as onsets the $n-k$ pulses 
that are not onsets in $R$. The study of the complementary sets of sets of intervals in the context of pitch (scales and chords) has received a lot of attention in music theory [6]. Rhythm, on the other hand, has been explored little from its complementarity aspects. Consider the Cuban cinquillo rhythm given by $[x \cdot x \times \cdot \times x \cdot]$. Its complementary rhythm is $[\cdot x \cdot x \cdot x]$, which is a rotation of the famous Cuban tresillo rhythm given by $[\times \cdots \times \cdot \times \cdot]$ (Figure 2). Complementary sets have many applications such as the composition of rhythmic complementary canons [10]. Clough and Douthett [1] show the following:

Theorem 2 The complement of a Euclidean rhythm is Euclidean.

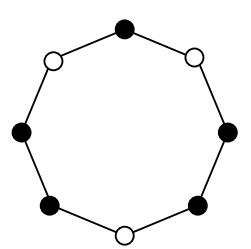

(a)

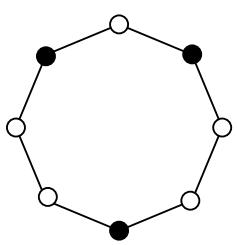

(b)

Figure 2: (a) The Cuban cinquillo (b) The complement of the Cuban cinquillo is a rotation of the Cuban tresillo.

\subsection{Concatenation}

The concatenation of two rhythms $R_{1}$ and $R_{2}$, denoted $R_{1} \oplus R_{2}$, is a new rhythm $R$ formed by the pulses of $R_{1}$ followed by those of $R_{2}$. For example, $E(3,7) \oplus E(4,6)=[\times \cdot \times \cdot \times \cdot \cdot] \oplus[\times \cdot \times \times \cdot$ $\times]=[\times \cdot \times \cdot \times \cdot \times \cdot \times x \cdot \times]$.

Theorem 3 For any Euclidean rhythm $E(k, n)$ with $1 \leq k \leq n$, and any natural number $c, E(c k, c n) \oplus$ $E(k, n)$ is a rotation of $E((c+1) k,(c+1) n)$.

In general it is not true that the concatenation of two arbitrary Euclidean rhythms is Euclidean. The concatenation of rhythms $E(3,7) \oplus E(4,6)$ is $[\times \cdot \times \cdot \times \cdots \times \cdot \times \times \cdot \times]$, which is not equal to $E(7,13)$ or to any of its rotations.

\subsection{Alternation}

The alternation operation transforms every other onset of a rhythm $R$ into a silence. Every rhythm has two alternations: an even alternation, where we keep the first onset, and change the second into a silence; and an odd alternation, where we keep the second onset, and change the first into a silence. We can show the following:

Theorem 4 The even and odd alternations of the Euclidean rhythm $E(2 k, 2 n)$ are rotations of $E(k, 2 n)$ for any $1 \leq k \leq n$.

\section{References}

[1] J. Clough and J. Douthett. Maximally even sets. Journal of Music Theory, 35:93-173, 1991.

[2] E. D. Demaine, F. Gomez-Martin, H. Meijer, D. Rappaport, P. Taslakian, G. T. Toussaint, T. Winograd, and D. R. Wood. The distance geometry of music. Submitted, 2006. 
[3] J. Fauvel, R. Flood, and R. Wilson. Music and mathematics: from Pythagoras to fractals. Oxford University Press, USA, 2003.

[4] L. Harkleroad. The math behind the music. Cambridge University Press, Cambridge, 2006.

[5] R. Hitt and X.-M. Zhang. Dynamic geometry of polygons. Elemente der Mathematik, 56:21-37, 2001.

[6] R. D. Morris. Pitch-class complementation and its generalizations. Journal of Music Theory, 34(2):175-245, Autumn 1990.

[7] J. Rahn. Turning the analysis around: African-derived rhythms and Europe-derived music theory. Black Music Research Journal, 16(1):71-89, 1996.

[8] I. J. Schoenberg. Mathematical Time Exposures. Mathematical Association of America, Washington, DC, 1982.

[9] G. T. Toussaint. A mathematical analysis of African, Brazilian, and Cuban clave rhythms. In Proceedings of BRIDGES: Mathematical Connections in Art, Music and Science, pages 157-168, Towson University, Towson, Maryland, U.S.A., July 27-29 2002.

[10] D. T. Vusa. Supplementary sets and regular complementary unending canons (part one). Perspectives of New Music, 29(2):22-49, Summer 1991. 